\title{
Modified ratio cum product estimators for estimation of finite population mean with known correlation coefficient
}

\begin{abstract}
In this paper, a modified ratio cum product estimator for the estimation of finite population mean of the study variable using the known correlation coefficient of the auxiliary variable is introduced. The bias and mean squared error of the proposed estimator are also obtained. The relative performance of the proposed estimator along with some existing estimators is accessed for certain labeled and natural populations. The results show that the proposed estimator is to be more efficient than the existing estimators.
\end{abstract}

Keywords: bias, mean squared error, natural population, simple random sampling, linear regression estimator
Volume 4 Issue 6 - 2016

Jambulingam Subramani, Master Ajith S

Department of Statistics, Ramanujan School of Mathematical

Sciences, Pondicherry University, India

Correspondence: Jambulingam Subramani, Department of Statistics, Ramanujan School of Mathematical Sciences, Pondicherry University, RV Nagar, Kalapet, Pondicherry, India,

Email drjsubramani@yahoo.co.in

Received: September 26, 2016 | Published: November 15, 2016

\section{Introduction}

In sampling theory, a wide variety of techniques is used to obtain efficient estimators for the population mean. The commonly used method to obtain the estimator for population mean is simple random sampling without replacement (SRSWOR) when there is no auxiliary variable available. There are methods that use the auxiliary information of the study characteristics. If there exists an auxiliary variable $\mathrm{X}$ which is correlated with the study variable $\mathrm{Y}$, then a number of estimators such as ratio, product, modified ratio, modified product, regression estimators and their modifications are widely available for estimation of population mean of the study variable $\mathrm{Y}$.

Consider a finite population $U=\left\{U_{1}, U_{2}, U_{3} \ldots U_{N}\right\}$ of $\mathrm{N}$ distinct and identifiable units. Let $Y$ be the study variable which takes the values $Y=\left\{Y_{1}, Y_{2}, Y_{3}, \ldots Y_{N}\right\}$. Here the problem is to estimate the population mean $\bar{Y}=\frac{1}{N} \sum_{i=1}^{N} Y_{i}$ on the basis of a random sample selected from the population $U$.

Before discussing further the various estimators, the notations to be used in this article are listed here.

$\begin{array}{lll}\mathrm{N} & - & \text { Population size } \\ \mathrm{n} & - & \text { Sample size } \\ f=\frac{n}{N} & - & \text { Sampling fraction } \\ \mathrm{Y} & - & \text { Study variable } \\ \mathrm{X} & - & \text { Auxiliary variable } \\ \bar{X}, \bar{Y} & - & \text { Population means } \\ \bar{x}, \bar{y} & - & \text { Sample means }\end{array}$

$\begin{array}{lll}S_{x}, S_{y} & - & \text { Population standard deviations } \\ S_{x}, S_{y} & - & \text { Sample standard deviations } \\ C_{x}, C_{y} & - & \text { Coefficient of variations } \\ \rho & - & \text { Correlation coefficient between x and y } \\ \beta_{1} & - & \text { Coefficient of skewness } \\ \beta_{2} & - & \text { Coefficient of kurtosis } \\ \mathrm{B}(.) & - & \text { Bias of estimators } \\ \operatorname{MSE}(.) & - & \text { Mean squared error of estimators }\end{array}$

In simple random sampling without replacement, the estimator $\bar{y}_{s r s}$ is an unbiased estimator for the population mean $\bar{Y}$ and its variance is given by

$$
V\left(\bar{y}_{s r s}\right)=\delta S_{y}^{2}
$$

Where $\delta=\left(\frac{1-f}{n}\right)$

Cochran, ${ }^{1}$ use auxiliary information for the estimation of population mean of the variable under study and proposed the ratio estimator of the population mean $\bar{Y}$ of the study variable, 


$$
\hat{Y}_{R}=\frac{\bar{y}}{\bar{x}} \bar{X}=\hat{R} \bar{X}
$$

The bias and mean squared error of the ratio estimator are given by

$$
\begin{gathered}
B\left(\overline{\bar{Y}}_{R}\right)=\delta \bar{Y}\left[C_{x}^{2}-\rho C_{x} C_{y}\right] \\
\operatorname{MSE}\left(\overline{\bar{Y}}_{R}\right)=\delta \bar{Y}^{2}\left[C_{y}^{2}+C_{x}^{2}-2 \rho C_{x} C_{y}\right]
\end{gathered}
$$

The linear regression estimator and its variance are given by

$$
\begin{gathered}
\bar{y}_{l r}=\bar{y}+b(\bar{X}-\bar{x}) \\
V\left(\bar{y}_{l r}\right)=\delta S_{y}^{2}\left(1-\rho^{2}\right)
\end{gathered}
$$

where $\mathrm{b}$ is the regression coefficient $\mathrm{Y}$ on $\mathrm{X}$

Murthy ${ }^{2}$ proposed the product estimator to estimate the population mean of the study variable when there is a negative correlation between the study variable $\mathrm{Y}$ and auxiliary variable $\mathrm{X}$ as

$$
\overline{\bar{Y}}_{p}=\bar{y}_{\bar{X}}^{\bar{x}}
$$

The bias and the mean squared error of the product estimator are given by

$$
\begin{gathered}
B\left(\bar{Y}_{p}\right)=\delta \bar{Y}\left[\rho C_{x} C_{y}\right] \\
M S E\left(\hat{\bar{Y}}_{p}\right)=\delta \bar{Y}^{2}\left[C_{y}^{2}+C_{x}^{2}+2 \rho C_{x} C_{y}\right]
\end{gathered}
$$

Singh and Tailor ${ }^{3}$ introduced the modified ratio estimator for the population mean with known population correlation coefficient $\rho$ of the auxiliary variable and is given by

$$
\hat{\bar{Y}}_{M R}=\bar{y}\left(\frac{\bar{X}+\rho}{\bar{x}+\rho}\right)
$$

The bias and mean squared error of this modified ratio estimator are given by

$$
\begin{gathered}
B\left(\hat{\bar{Y}}_{M R}\right)=\delta \bar{Y}\left[\theta^{2} C_{x}^{2}-\theta \rho C_{x} C_{y}\right] \\
\operatorname{MSE}\left(\hat{\bar{Y}}_{M R}\right)=\delta \bar{Y}^{2}\left[C_{y}^{2}+\theta^{2} C_{x}^{2}-2 \theta \rho C_{x} C_{y}\right]
\end{gathered}
$$

where $\theta=\frac{\bar{X}}{\bar{X}+\rho}$
The modified product estimator with known correlation coefficient of the auxiliary variable when there is a negative correlation between the study variable $\mathrm{Y}$ and auxiliary variable $\mathrm{X}$ is given as

$$
\hat{\bar{Y}}_{M p}=\bar{y}\left(\frac{\bar{x}+\rho}{\bar{X}+\rho}\right)
$$

The bias and mean squared error of the modified product estimator are given by

$$
\begin{gathered}
B\left(\hat{\bar{Y}}_{M p}\right)=\delta \bar{Y}\left[\theta \rho C_{x} C_{y}\right] \\
\operatorname{MSE}\left(\hat{\bar{Y}}_{M p}\right)=\delta \hat{\bar{Y}}\left[C_{y}^{2}+\theta^{2} C_{x}^{2}+2 \theta \rho C_{x} C_{y}\right]
\end{gathered}
$$

where $\theta=\frac{\bar{X}}{\bar{X}+\rho}$

In literature, several estimators are available with auxiliary variables. However the problem is that the best estimator in terms of bias and efficiency are not fully addressed. In this paper, we attempt to solve such type of problems. The existing estimators are biased but the percentage relative efficiency is better than that of simple random sampling, ratio and product estimators. These points are motivated us to introduce a new class of improved ratio cum product estimators for the estimation of the population mean of the study variable.

\section{Proposed estimators}

For estimating population mean $\bar{Y}$ we have proposed a class of ratio cum product estimators ${ }^{4}$ for the population mean by using the known population correlation coefficient of the auxiliary variable and is given by

$$
\hat{\bar{Y}}_{p r}=\alpha \lambda_{1} \bar{y}\left(\frac{\bar{X}+\rho}{\bar{x}+\rho}\right)+(1-\alpha) \lambda_{2} \bar{y}\left(\frac{\bar{x}+\rho}{\bar{X}+\rho}\right)
$$

Here, $\lambda_{1}=\frac{S_{y}}{S_{y}+\gamma_{1} C_{y}}$ and $\lambda_{2}=\frac{S_{y}}{S_{y}+\gamma_{2} C_{y}}$,

$$
\gamma_{2}=B\left(\hat{\bar{Y}}_{M p}\right)
$$

\section{Bias and mean squared error of the proposed estimators}

The detailed derivation of the bias and mean squared error are given in the appendix whereas the procedures to obtain the bias and mean squared error of the proposed estimators are briefly outlined below: 
Consider, $e_{\theta}=\frac{\bar{y}-\bar{Y}}{\bar{Y}}, e_{1}=\frac{\bar{x}-\bar{X}}{\bar{X}} \quad \theta=\frac{\bar{X}}{\bar{X}+\rho}$

$$
\begin{gathered}
E\left(e_{\theta}\right)=E\left(e_{1}\right)=0 \\
E\left(e_{0}^{2}\right)=\delta \bar{Y}^{2} C_{y}^{2}, E\left(e_{1}^{2}\right)=\delta \bar{X}^{2} C_{x}^{2}, E\left(e_{0} e_{1}\right)=\delta \rho C_{x} C_{y}
\end{gathered}
$$

Substitute these values in equation (9) and neglecting the high order expressions, we get

$$
\begin{gathered}
B\left(\hat{\bar{Y}}_{\mathrm{Pr}}\right)=E\left(\hat{\bar{Y}}_{\mathrm{Pr}}-\bar{Y}\right) \\
B\left(\hat{\bar{Y}}_{\mathrm{Pr}}\right)=\bar{Y}\left(\alpha \lambda_{1}+(1-\alpha) \lambda_{2}-1\right)+\delta \bar{Y}\left\{\alpha \lambda_{1} \theta^{2} C_{x}^{2}-\theta \rho C_{x} C_{y}\left(\alpha \lambda_{1}-(1-\alpha) \lambda_{2}\right)\right\} \\
M S E\left(\hat{\bar{Y}}_{\mathrm{Pr}}\right)=\bar{Y}^{2}(A-1)^{2}+\delta \bar{Y}^{2}\left\{C_{y}{ }^{2}\left(\alpha \lambda_{1}+(1-\alpha) \lambda_{2}\right)^{2}+\theta^{2} C_{x}{ }^{2}\left(3 \alpha^{2} \lambda_{1}{ }^{2}+(1+\alpha)^{2} \lambda_{2}{ }^{2}-2 \alpha \lambda_{1}\right)+2 \theta \rho C_{x} C_{y}\left(\alpha \lambda_{1}-(1-\alpha) \lambda_{2}\right)-2\left(\alpha^{2} \lambda_{1}{ }^{2}-(1-\alpha)^{2} \lambda_{2}{ }^{2}\right)\right\} \\
M S E\left(\hat{\bar{Y}}_{\mathrm{Pr}}\right)=\bar{Y}^{2}(A-1)^{2}+\delta \bar{Y}^{2}\left\{A^{2} C_{y}{ }^{2}+\theta^{2} C_{x}{ }^{2}\left(A^{2}+(A+B)(B-1)\right)-2 \theta \rho C_{x} C_{y} B(2 A-1)\right\}
\end{gathered}
$$

where $A=\left(\alpha \lambda_{1}+(1-\alpha) \lambda_{2}\right), B=\left(\alpha \lambda_{1}-(1-\alpha) \lambda_{2}\right)$ and $\theta=\frac{\bar{X}}{\bar{X}+\rho}$

The optimal value of $\alpha$ is determined by minimizing the MSE $\left(\hat{Y}_{p r}\right)$ with respect to $\alpha$. For this differentiate MSE with respect to $\alpha$ and equate to zero. ${ }^{5}$

$$
\begin{gathered}
\frac{\partial M S E}{\partial \alpha}=0, \text { and we get the value of } \alpha, \text { as } \\
\alpha=\frac{\left(\lambda_{2}-1\right)\left(\lambda_{2}-\lambda_{1}\right)-\delta\left[C_{y}{ }^{2} \lambda_{2}\left(\lambda_{1}-\lambda_{2}\right)-\theta^{2} C_{x}{ }^{2}\left(\lambda_{1}+\lambda_{2}{ }^{2}\right)+\theta \rho C_{x} C_{y}\left(\lambda_{1}+\lambda_{2}-4 \lambda_{2}{ }^{2}\right)\right]}{\left(\lambda_{1}-\lambda_{2}\right)^{2}+\delta\left[\left(\lambda_{1}-\lambda_{2}\right)^{2} C_{y}{ }^{2}+\theta^{2} C_{x}{ }^{2}\left(3 \lambda_{1}{ }^{2}+\lambda_{2}{ }^{2}\right) 4 \theta \rho C_{x} C_{y}\left(\lambda_{2}{ }^{2}-\lambda_{1}{ }^{2}\right)\right]}
\end{gathered}
$$

\section{Efficiency comparison}

The efficiencies of the proposed estimators with that of the existing estimators are obtained algebraically and are as follows:

\section{Comparison of proposed estimator and simple random sampling (SRSWOR) estimator}

The proposed estimator is more efficient than simple random sampling estimator,

$$
\begin{gathered}
V\left(\bar{y}_{l r}\right) \geq \operatorname{MSE}\left(\hat{\bar{Y}}_{\mathrm{Pr}}\right) \text { if } \\
C_{y}^{2} \geq \frac{\left\{(A-1)^{2}+\delta^{2}\left\{\theta^{2} C_{x}^{2}\left(A^{2}+(A+B)(B-1)\right)-2 \theta \rho C_{x} C_{y} B(2 A-1)\right\}\right\}}{\delta^{2}\left(1-A^{2}\right)}
\end{gathered}
$$

\section{Comparison of proposed estimator and linear regression estimator}

The proposed estimator is more efficient than linear regression estimator,

$$
V\left(\bar{y}_{l r}\right) \geq \operatorname{MSE}\left(\hat{\bar{Y}}_{\operatorname{Pr}}\right) \text { if }
$$

$C_{y}^{2} \geq \frac{\left\{(A-1)^{2}+\delta\left[\theta^{2} C_{x}^{2}\left(A^{2}+(A+B)(B-1)\right)-2 \theta C_{x} C_{y}(B(2 A-1)-1)\right]\right\}}{\delta\left(1-\rho^{2}-A^{2}\right)}$

Comparison of proposed estimator and ratio estimator

The proposed estimator is more efficient than ratio estimator 


$$
\begin{gathered}
\operatorname{MSE}\left(\hat{\bar{Y}}_{P}\right) \geq \operatorname{MSE}\left(\hat{\bar{Y}}_{\mathrm{Pr}}\right) \text { if } \\
C_{y}^{2} \geq \frac{\left\{(A-1)^{2}+\delta\left\{C_{x}^{2}\left(\theta^{2}\left(A^{2}+(A+B)(B-1)\right)-1\right)-2 \rho C_{x} C_{y}(\theta B(2 A-1)-1)+1\right\}\right\}}{\delta\left(1-A^{2}\right)}
\end{gathered}
$$

\section{Comparison of proposed estimator and product estimator}

The proposed estimator is more efficient than ratio estimator, ${ }^{6}$

$$
\begin{gathered}
\operatorname{MSE}\left(\hat{\bar{Y}}_{P}\right) \geq \operatorname{MSE}\left(\hat{\bar{Y}}_{\mathrm{Pr}}\right) \text { if } \\
C_{y}^{2} \geq \frac{\left\{(A-1)^{2}+\delta\left\{C_{x}^{2}\left(\theta^{2}\left(A^{2}+(A+B)(B-1)\right)-1\right)-2 \rho C_{x} C_{y}(\theta B(2 A-1)+1)\right\}\right\}}{\delta\left(1-A^{2}\right)}
\end{gathered}
$$

\section{Comparison of proposed estimator and modified ratio estimator}

The proposed estimator is more efficient than modified ratio estimator ${ }^{7}$

$$
\begin{gathered}
\operatorname{MSE}\left(\hat{\bar{Y}}_{\text {MR }}\right) \geq \operatorname{MSE}\left(\hat{\bar{Y}}_{\mathrm{Pr}}\right) \\
C_{y}^{2} \geq \frac{\left\{(A-1)^{2}+\delta\left\{\theta^{2} C_{x}^{2}\left(A^{2}+(A+B)(B-1)-1\right)-2 \theta \rho C_{x} C_{y}(\theta B(A-1)-1)-1\right\}\right\}}{\delta\left(1-A^{2}\right)}
\end{gathered}
$$

\section{Comparison of proposed estimator and modified product estimator}

The proposed estimator is more efficient than modified product estimator,

$$
\begin{gathered}
\operatorname{MSE}\left(\hat{\bar{Y}}_{M p}\right) \geq \operatorname{MSE}\left(\hat{\bar{Y}}_{\mathrm{Pr}}\right) \text { if } \\
C_{y}^{2} \geq \frac{\left\{(A-1)^{2}+\delta\left\{\theta^{2} C_{x}^{2}\left(A^{2}+(A+B)(B-1)-1\right)-2 \theta \rho C_{x} C_{y}(B(2 A-1)-1)\right\}\right\}}{\delta\left(1-A^{2}\right)}
\end{gathered}
$$

\section{Numerical study}

In this section, we consider the four natural populations population 1 Khoshnevisan et al., ${ }^{8}$ Population 2 Cochran ${ }^{9}$ (page 325) population 3 and 4 Singh and Chaudhary, ${ }^{10}$ (page 177) and are used to compare the percentage relative efficiency of proposed estimator with that of the existing estimators such as SRSWOR sample mean, linear regression estimator, ratio estimator, product estimator, modified ratio estimators, and modified product estimators.

\section{Conclusion}

We have proposed a class of modified ratio cum product estimators for finite population ${ }^{11}$ mean of the study variable $\mathrm{Y}$ with known correlation coefficient of the auxiliary variable $\mathrm{X}$. The bias and mean squared error of the proposed estimators are obtained and compared with that of the simple random sampling without replacement, regression, ratio, product, modified ratio, modified product estimators by both algebraically and numerically. We support this theoretical result with numerical examples. We have shown that the proposed estimator is more efficient than other existing estimators under the optimum values of $\alpha$. Table $1 \& 2$ shows that the bias and MSE of the proposed estimators are smaller than the other competing estimators. Table 3 shows that the percentage relative efficiency of the proposed estimator with respect to the existing estimators, 
Table I The computed values of constants and parameters from different populations

\begin{tabular}{lllll}
\hline Parameters & Population I & Population 2 & Population 3 & Population 4 \\
\hline $\mathrm{N}$ & 20 & 10 & 34 & 34 \\
$\mathrm{n}$ & 8 & 3 & 3 & 5 \\
$\bar{Y}$ & 19.55 & 101.1 & 856.4117 & 856.4117 \\
$\bar{X}$ & 18.8 & 58.8 & 208.8823 & 208.8823 \\
$\rho$ & -0.9199 & 0.6515 & 0.4491 & 0.4491 \\
& 6.9441 & 15.4448 & 733.1407 & 733.1407 \\
& 0.3552 & 0.1527 & 0.8561 & 0.8561 \\
& 7.4128 & 7.9414 & 150.5059 & 150.5059 \\
& 0.3943 & 0.1351 & 0.7205 & 0.7205 \\
& 3.0613 & 0.2363 & 2.9123 & 2.9123 \\
& 0.5473 & 2.2388 & 0.9781 & 0.9781 \\
& 1.0514 & 0.989 & 0.9978 & 0.9965 \\
& 0.4506 & 0.1072 & 625915 & 35.1319 \\
& -0.1986 & 0.3136 & 71.947 & 40.3832 \\
0.9774 & 0.9989 & 0.9319 & 0.9605 \\
1.0102 & 0.9969 & 0.9225 & 0.9549 \\
0.1055 & 0.8717 & 0.7614 & 0.7639 \\
\hline
\end{tabular}

Table 2 Bias and MSE of proposed and existing estimators from different population

\begin{tabular}{lllllllll}
\hline \multirow{2}{*}{ Estimator } & \multicolumn{2}{l}{ Population I } & \multicolumn{3}{c}{ Population 2 } & \multicolumn{2}{c}{ Population 3 } & \multicolumn{2}{c}{ Population 4 } \\
\cline { 2 - 9 } & Bias & MSE & Bias & MSE & Bias & MSE & Bias & MSE \\
\hline Proposed & $1.14 \mathrm{e}-06$ & 0.5463 & $1.13 \mathrm{e}-15$ & 31.9319 & -0.00274 & 109092.8 & -0.0015 & 66145.84 \\
$\bar{y}_{s r s}$ & - & 3.6166 & - & 55.6603 & - & 163356.4 & - & 91690.37 \\
$\bar{y}_{l r}$ & - & 0.5561 & - & 32.0343 & - & 130408.9 & - & 73197.27 \\
$\overline{\bar{Y}}_{R}$ & 0.4168 & 15.4595 & 0.1132 & 35.0447 & 63.0193 & 155580.6 & 35.3721 & 87325.9 \\
$\overline{\bar{Y}}_{p}$ & -0.1889 & 0.6869 & 0.3171 & 163.283 & 72.0984 & 402564.2 & 40.4681 & 225955.4 \\
$\overline{\bar{Y}}_{M R}$ & 0.4506 & 16.3099 & 0.1072 & 34.7991 & 62.5915 & 155359.1 & 35.1319 & 87201.54 \\
$\overline{\bar{Y}}_{M P}$ & -0.1986 & 0.7774 & 0.3163 & 161.6321 & 71.947 & 401824.2 & 40.3832 & 225540 \\
\hline
\end{tabular}

In fact, the PRE is ranging from

I. $\quad 138.6185$ to 661.9810 in case of SRSWOR sample mean

II. $\quad 100.3205$ to 119.4555 in case of Linear Regression Estimator

III. $\quad 109.7481$ to 2829.7520 in case of Ratio estimator

IV. $\quad 125.7468$ to 511.3465 in case of Product estimator

V. $\quad 108.9790$ to 2985.4080 in case of Modified Ratio estimator and

VI. $\quad 142.2864$ to 506.1764 in case of Product estimator

From this, we have observed that the proposed estimator is performed better than that of other existing estimators and hence we recommend the proposed estimators for the practical problems. 
Table 3 Percentage relative efficiency of the proposed estimator

\begin{tabular}{lllll}
\hline Estimators & Population & Population 2 & Population 3 & Population 4 \\
\hline $\bar{y}_{s r s}$ & 661.981 & 174.3092 & 149.6356 & 138.6185 \\
$\bar{y}_{l r}$ & 101.802 & 100.3205 & 119.4555 & 110.6604 \\
$\overline{\bar{Y}}_{R}$ & 2829.752 & 109.7481 & 142.5216 & 132.0283 \\
$\hat{\bar{Y}}_{p}$ & 125.7468 & 511.3465 & 368.7708 & 341.6196 \\
$\hat{\bar{Y}}_{M R}$ & 2985.408 & 108.979 & & 131.8322 \\
$\hat{\bar{Y}}_{M P}$ & 142.2864 & 506.1764 & 367.6544 & 340.9739 \\
\hline
\end{tabular}

\section{Acknowledgement}

None.

\section{Conflict of interest}

None.

\section{References}

1. Cochran WG. The estimation of the yields of the cereal experiments by sampling for the ratio of grain to total produce. The Journal of Agricultural Science. 1940;30(2):262-275.

2. Murthy MN. Product method of estimation. Sankhyā:. The Indian Journal of Statistics. 1964;26(1):69-74.

3. Singh HP, Tailor R. Use of known correlation coefficient in estimating the finite population means. Statistics in Transition. 2003;6(4):555-560.

4. Ekaette Inyang Enang, Victoria Matthew Akpan, Emmanuel John Ekpenyong. Alternative ratio estimator of population mean in simple random sampling, Journal of Mathematics Research. 2014;6(3).

5. Housila P Singh, Surya K Pal, Vishal Mehta. A generalized class of dual to product-cum-dual to ratiotype estimators of finite population mean in sample surveys. Appl Math Inf Sci Lett. 2016;4(1):25-33.

6. J Subramani G Kumarapandiyan. A class of almost unbiased modified ratio estimators for population mean with known population parameters. Elixir Statistics. 2012;44:7411-7415.

7. Murthy MN. Sampling theory and methods. Statistical Publishing Society, Calcutta, India. 1967.

8. Khoshnevisan M, Singh R, Chauhan P, et al. A general family of estimators for estimating population mean using known value of some population parameter(s). Far East Journal of Theoretical Statistics. 2007;22:181-191.

9. Cochran WG. Sampling Techniques. ( $3^{\text {rd }}$ edn), Wiley Eastern Limited, India. 1977;pp. 448.

10. Singh D, Chaudhary FS. Theory and analysis of sample survey designs. ( $1^{\text {st }}$ edn), New Age International Publisher, India, 1986;pp. 332.

11. Subramani J. "Generalized modified ratio estimator for estimation of finite population mean". Journal of Modern Applied Statistical Methods. 2013;12(2):pp.121-155. 